\title{
Effect of continuous positive airway pressure therapy on circadian patterns of cardiac repolarization in patients with obstructive sleep apnoea: data from a randomized trial
}

\author{
Christian Schlatzer ${ }^{1}$, Daniel J. Bratton ${ }^{1}$, Esther I. Schwarz ${ }^{1}$, Thomas Gaisl ${ }^{1}$, Justin C. T. Pepperell ${ }^{2}$, John R. \\ Stradling, ${ }^{2}$ Malcolm Kohler ${ }^{1,3}$ \\ ${ }^{1}$ Sleep Disorders Center and Pulmonary Division, University Hospital Zurich, Zurich, Switzerland; ${ }^{2}$ Oxford Centre for Respiratory Medicine and \\ NIHR Oxford Biomedical Research Centre, Churchill Hospital, Oxford, UK; ${ }^{3}$ Zurich Center for Interdisciplinary Sleep Research, University of \\ Zurich, Zurich, Switzerland \\ Contributions: (I) Conception and design: C Schlatzer, JR Stradling, M Kohler, DJ Bratton; (II) Administrative support: All authors; (III) Provision \\ of study materials or patients: JC Pepperell, JR Stradling; (IV) Collection and assembly of data: C Schlatzer, JR Stradling, JC Pepperell; (V) Data \\ analysis and interpretation: All authors; (VI) Manuscript writing: All authors; (VII) Final approval of manuscript: All authors. \\ Correspondence to: Dr. Christian Schlatzer. Sleep Disorders Center and Pulmonary Division, University Hospital Zurich, Raemistrasse 100,8091 \\ Zurich, Switzerland. Email: christianschlatzer@gmail.com.
}

Background: Obstructive sleep apnoea (OSA) has been proposed as an independent risk factor for sudden cardiac death (SCD). This study takes advantage of a previous randomized trial and seeks to evaluate circadian patterns of the QTc-interval, a marker of cardiac repolarization and biomarker for SCD, in patients with OSA. We hypothesized that patients with OSA would exhibit longest QTc during the night-time and that continuous positive airway pressure (CPAP) therapy would reverse this.

Methods: One hundred eighteen patients diagnosed with moderate-to-severe OSA were randomized to receive therapeutic or subtherapeutic CPAP for 4 weeks. Of these, 84 had full 24 h-Holter monitoring data at baseline and follow-up. Weighted means of all QTc-intervals were analysed over $24 \mathrm{~h}$, during four timeperiods (12 pm-6 am, 6 am-12 am, 12 am-6 pm, 6 pm-12 pm) as well as during each individual hour. A twosided $\mathrm{P}$ value $<0.05$ was considered to be of statistical significance.

Results: QTc-intervals at baseline [mean (SD) over 24 h: $407.8 \mathrm{~ms}$ (36.6)] were highest from $6 \mathrm{pm}-12 \mathrm{pm}$ [411.7 ms (42.0)] and shortest from $6 \mathrm{am}-12 \mathrm{am}$ [405.4 ms (39.5)]. Overall $24 \mathrm{~h}$ CPAP treatment effect on QTc was $-11.3 \mathrm{~ms}$ [95\% confidence interval $(\mathrm{CI}),-22.1$ to $-0.6 ; \mathrm{P}=0.039]$ and was estimated to be greater from 6 pm-12 pm than from 12 pm-6 am (P=0.068). The CPAP treatment effect on QTc was driven by those patients in the highest QTc decile at baseline (all $>430 \mathrm{~ms}$ ). In these patients, CPAP led to reductions in QTc, allowing reclassification into lower risk-associated values of QTc (<430 ms).

Conclusions: In this exploratory study, CPAP treatment led to an overall reduction in the QTc-interval compared with subtherapeutic CPAP. This reduction seems more pronounced during evening hours and in patients with a QTc above $430 \mathrm{~ms}$.

Keywords: Obstructive sleep apnoea (OSA); sudden cardiac death (SCD); repolarization; QTc

Submitted Jan 12, 2018. Accepted for publication May 08, 2018.

doi: $10.21037 /$ jtd.2018.07.17

View this article at: http://dx.doi.org/10.21037/jtd.2018.07.17 


\section{Introduction}

Obstructive sleep apnoea (OSA) is a very common sleeprelated breathing disorder with estimated prevalence rates in the general population ranging from $2 \%$ to $25 \%$ in women and from $7 \%$ to $50 \%$ in men, when a cutoff of the apnoea-hypopnoea-index (AHI) $\geq 15$ per $\mathrm{h}$ is applied, representing moderate-to-severe OSA $(1,2)$. During sleep, repetitive interruption of ventilation, caused by partial or complete collapse of the pharynx, results in apnoea and arousals from sleep, thus leading to excessive daytime sleepiness, increased accident rates, elevated blood pressure and vascular dysfunction (3). OSA has been found to be independently associated with sudden cardiac death (SCD) in a large cohort study (4). It remains challenging to predict SCD, but one established marker for SCD risk stratification is the heart rate corrected QTc-interval, a marker of ventricular repolarization (5). It encompasses ventricular de- and repolarization, starting with the onset of the Q-wave and ending with the end of the T-wave. If prolonged, it is associated with torsade-de-pointes ventricular tachyarrhythmia, and SCD. Patients with OSA seem to be at greatest risk for SCD during sleeping hours, as opposed to patients without OSA (and the general population), in whom SCD is least likely to occur during that time period (6). This observation could in part be due to particularly long QTc-intervals during the night in OSA patients and is possibly reversible through continuous positive airway pressure (CPAP) therapy.

We have previously shown a clinically significant prolongation of the QTc-interval after a 2-week withdrawal of CPAP therapy in patients with OSA (7). A limitation of our previous study was that the QTc data had been derived from a standard resting 12-lead-electrocardiogram (ECG), thus not allowing a detailed analysis of day/night patterns of repolarization. Therefore, in the present study we sought to evaluate the effects of CPAP therapy on QTc-duration by an analysis of 24-h-Holter-ECG data collected in a previous RCT (8).

We hypothesized that QTc in moderate-to-severe OSA patients at baseline would be highest during sleeping hours not only because this is found in the general population $(9,10)$ but also because OSA may further lengthen it. If this is the case, CPAP would be expected to partially reverse this.

\section{Methods}

\section{Study design}

Patients with possible OSA were referred to the Sleep and Respiratory Trials Unit, Oxford Centre for Respiratory Medicine, Oxford, UK and assessed for eligibility for a parallel, randomized, double-blind trial. The trial from which the data were drawn was previously published in 2002 (8) and was approved by the central Oxford research ethics committee (Oxford, UK; COREC No. 96.127).

\section{Study participants}

Male patients aged between 30 and 75 years with excessive subjective daytime sleepiness [Epworth sleepiness scale (ESS) >9] (11) and proven OSA with $>10 \mathrm{dips} / \mathrm{h}$ of $>4 \%$ oxygen desaturation (ODI $>10)$ were eligible for the trial. We did not include patients who preferred alternative treatments such as weight loss or tonsillectomy, or if they required urgent CPAP treatment due to respiratory failure or sleepiness-related vocational problems. Cardiovascular disease was not an exclusion criterion. All participants gave written informed consent.

\section{Sleep study}

A one-night in-hospital polygraphic (PG) sleep study was performed to diagnose OSA. The sleep study results were scored automatically, and subsequently manually reviewed for accuracy. An ODI of 10/h due to obstructive apnoeas was used as a cutoff, above which OSA was diagnosed.

\section{Electrocardiography}

Twenty-four-h-Holter electrocardiography was applied during the hospital assessments in the early afternoon. A commercially available (Cardioscan DL700; Breamar Inc., Bramwell, MN, USA) 3-channel-Holter recorder was used. The devices were then worn for the following $24 \mathrm{~h}$ during normal daily activities. Data were analysed using the Century 3000 system (UK Medical, Sheffield, UK). The QT-interval was defined as the time-interval from the onset of the QRS complex to the cutting point of the tangent to the downward slope of the $\mathrm{T}$ wave and the isoelectric line. 
The QT-interval was then corrected for heart rate using Bazett's formula (12).

\section{CPAP therapy and randomization}

After the first 24 h-ECG monitoring at baseline, participants were randomly assigned to either receive therapeutic or subtherapeutic CPAP by a series of presealed and numbered opaque envelopes and attended for a second in-hospital sleep study. A specialized nurse assigned participants to treatment groups and did not take part in the outcome analysis. The outcome assessors were not aware of the allocation of patients and were not involved in the setup and maintenance of CPAP devices. Sullivan Autoset-T autoadjusting (ResMed, Abingdon, UK) CPAP machines were used in both groups. Subtherapeutic CPAP, at too low a pressure to splint open the pharynx, was obtained by setting the CPAP machine to its lowest pressure, connecting a flow restrictor to the main tubing, and creating six extra 4 $\mathrm{mm}$ holes in the collar of the main tubing at the end of the mask, to further drop the pressure (to $<1 \mathrm{cmH}_{2} \mathrm{O}$ ) and thus prevent rebreathing of carbon dioxide. Further details are available in the prior publication (8).

\section{Outcomes}

The primary endpoint of interest in this analysis was the CPAP treatment effect on the overall $24 \mathrm{~h}$-QTc-interval. Secondary endpoints were baseline QTc-intervals and the CPAP treatment effect on QTc during four time periods (night from 12 pm-6 am, morning from 6 am-12 am, afternoon from 12 am-6 pm and evening from 6 pm-12 pm) and during each individual hour.

\section{Data analysis}

Continuous variables were summarized as mean (SD). For each patient the mean QTc-interval in each time period of interest was calculated using a weighted mean with weights defined as the number of beats recorded in each hour. Mean QTc-intervals at baseline were summarized in the time periods defined above and compared using multilevel mixed-effects regression adjusting for time period and including a patient-specific random intercept.

Mean 24 h-intervals were compared between treatment groups using multiple linear regression. In the time periods defined above, outcomes were compared between treatment groups using multi-level mixed-effects regression including a treatment $\mathrm{x}$ time-period interaction and a patient-specific random intercept. Global tests for interaction were used to determine whether the effect of CPAP varied between each time-interval. All analyses were adjusted for the corresponding interval measured over $24 \mathrm{~h}$ at baseline and residuals were investigated for possible outliers.

The interaction between baseline mean $24 \mathrm{~h}$ QTc, ODI and CPAP usage with treatment effect on mean $24 \mathrm{~h}$ QTc was assessed using fractional polynomials, as described by Royston and Sauerbrei (13). The results of the modelling procedure were checked by estimating the treatment effect of CPAP at each decile of baseline mean $24 \mathrm{~h}-\mathrm{QT}$. A two-sided $\mathrm{P}$ value $<0.05$ was considered to be of statistical significance.

\section{Results}

\section{Trial profile and patient characteristics}

Figure 1 shows the trial profile. Of the 339 eligible participants initially approached, 155 did not consent. Another 25 participants chose an alternative treatment, 11 needed urgent CPAP treatment, 11 did not attend the first study day and 19 had inadequate baseline blood pressure, which was an exclusion criterion for the previously published trial (8). The remaining 118 participants were randomized to either receive therapeutic or subtherapeutic CPAP. In the therapeutic CPAP group, 2 patients discontinued CPAP, 4 did not attend for follow-up and 11 had no, or inadequate (artifacts), ECG data at follow-up. In the subtherapeutic CPAP group, 2 discontinued CPAP, 6 did not attend for follow-up and 13 had no, or inadequate (artifacts), ECG data at follow-up. Thus data from 84 participants were available for analysis. Table 1 shows baseline characteristics of these participants, which were similar between treatment groups. There was no evidence of any significant difference in treatment effects of CPAP on OSA severity, subjective and objective sleepiness in our subset of patients when compared to the original trial (8).

\section{Baseline analysis}

In both groups combined, mean QTc over $24 \mathrm{~h}$ was $407.8 \mathrm{~ms}$ (36.6). Mean baseline QTc-intervals during four time periods are shown in Table 2 and during each individual hour in Figure 2. There was evidence of a difference in mean QTc between the four time periods (global test for difference $\mathrm{P}=0.005$ ) with $\mathrm{QTc}$-interval being longest during 


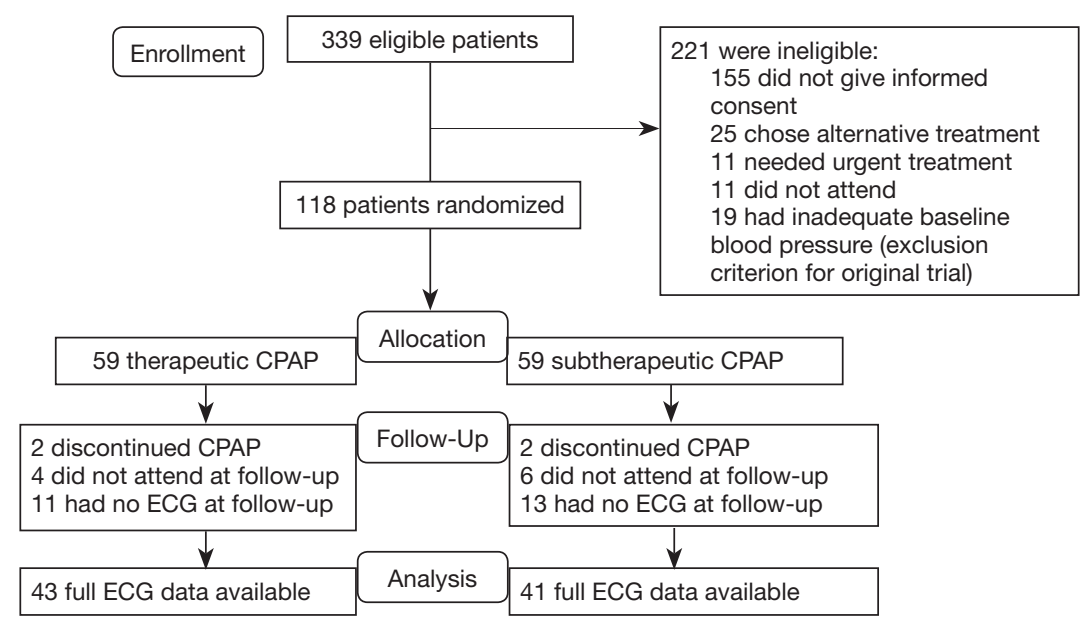

Figure 1 Study flow.

Table 1 Baseline characteristics

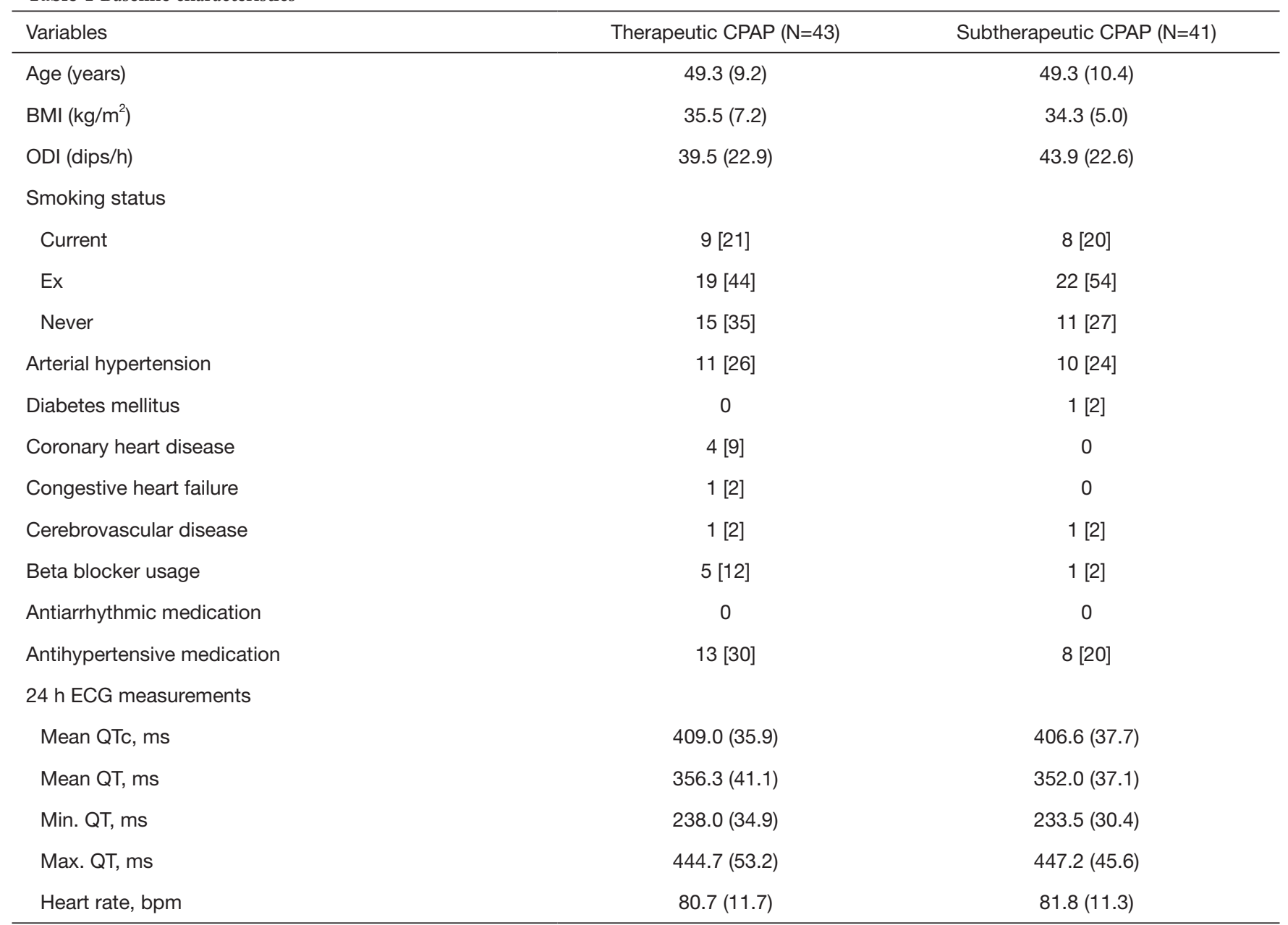

Data are presented as means (SD) or $\mathrm{n}(\%)$ as appropriate. BMI, body mass index; ECG, electrocardiogram; ODI, oxygen desaturation index; QT, time interval of cardiac repolarization in ms; QTc, time interval of cardiac repolarization in ms, corrected for heart rate. 
Table 2 Circadian effects on baseline QTc-intervals in both groups combined

\begin{tabular}{lcc}
\hline Time period & Mean QTc, ms & Global test for difference \\
\hline $12 \mathrm{pm}-6 \mathrm{am}$ & $405.5(38.4)$ & \\
$6 \mathrm{am}-12 \mathrm{am}$ & $405.4(39.5)$ & $\mathrm{P}=0.005$ \\
$12 \mathrm{am}-6 \mathrm{pm}$ & $407.5(39.6)$ & \\
$6 \mathrm{pm}-12 \mathrm{pm}$ & $411.7(42.0)$ & \\
Total 24 h & $407.8(36.6)$ & - \\
\hline
\end{tabular}

Data are presented as means (SD). QTc, time interval of cardiac repolarization in $\mathrm{ms}$, corrected for heart rate. evening hours with a mean of $411.7 \mathrm{~ms}$ (42.0). There was no correlation between baseline QTc and baseline severity of OSA (ODI) as a function of the $24 \mathrm{~h}$ period.

\section{Effect of CPAP on cardiac repolarization}

CPAP treatment effects on QTc are shown in Table 3. Therapeutic CPAP reduced mean QTc over 24 h by $-11.3 \mathrm{~ms}[95 \%$ confidence interval (CI), -22.1 to -0.6 ; $\mathrm{P}=0.039$ ] when compared with subtherapeutic CPAP. CPAP seemed to reduce QTc more during evening hours than during the rest of the day, although the test for treatment-

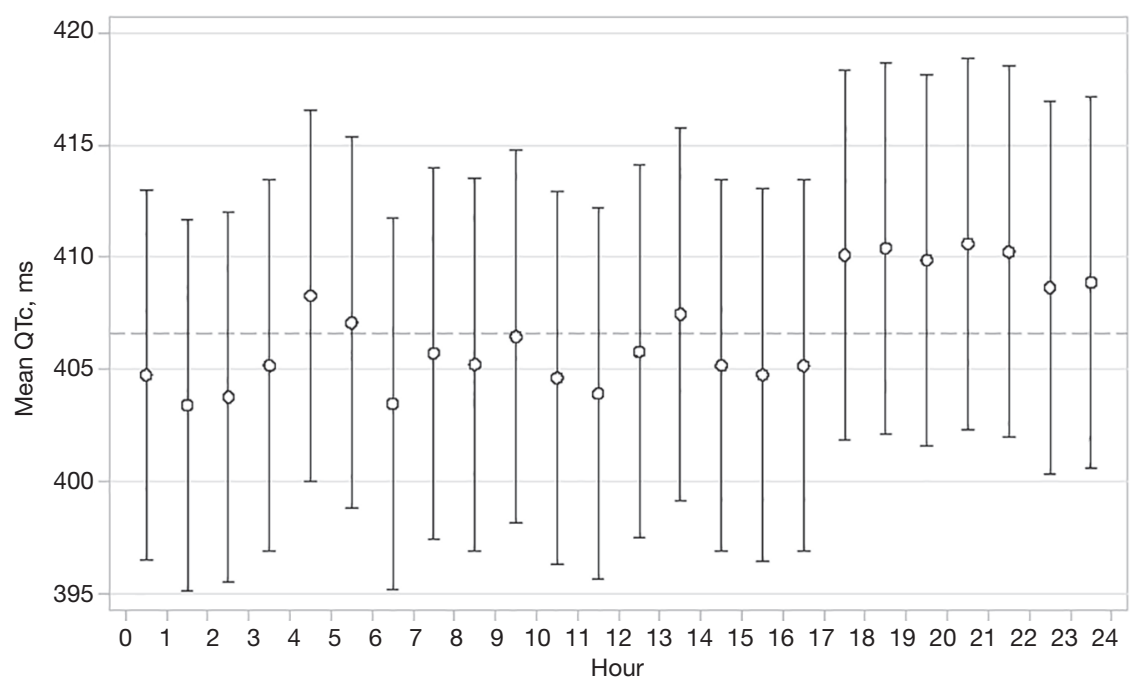

Figure 2 Baseline QTc-intervals in each individual hour in both groups. P value (H0: no change over time) $<0.001$. QTc, time interval of cardiac repolarization in $\mathrm{ms}$, corrected for heart rate.

Table 3 Effect of CPAP on mean $24 \mathrm{~h}$ QTc, and during the four time-periods

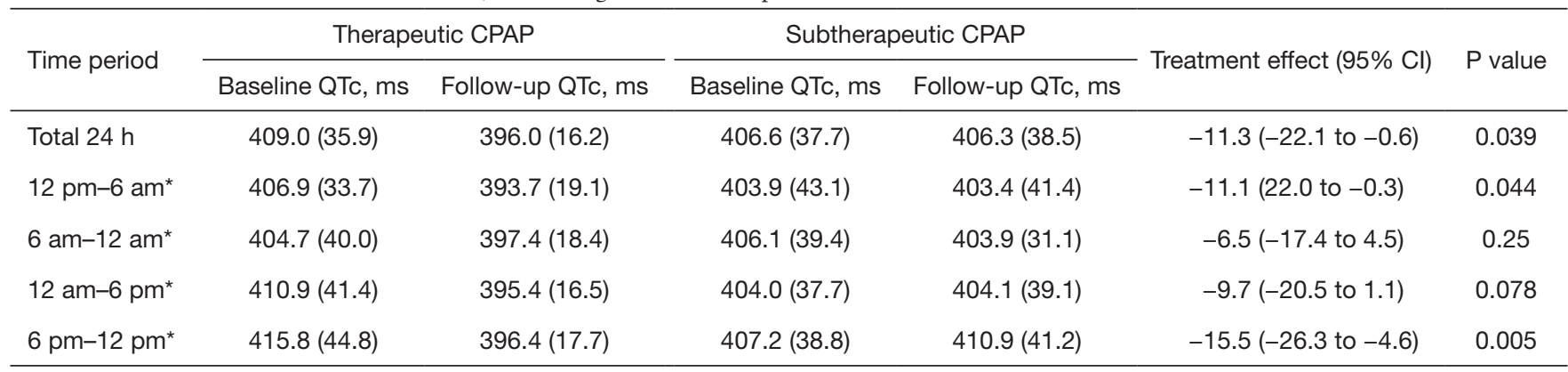

Data are presented as mean $(\mathrm{SD})$ or mean $(95 \% \mathrm{Cl}) .{ }^{*}$, test for treatment-time interaction: $\mathrm{P}=0.068$. QTc, time interval of cardiac repolarization in $\mathrm{ms}$, corrected for heart rate. 


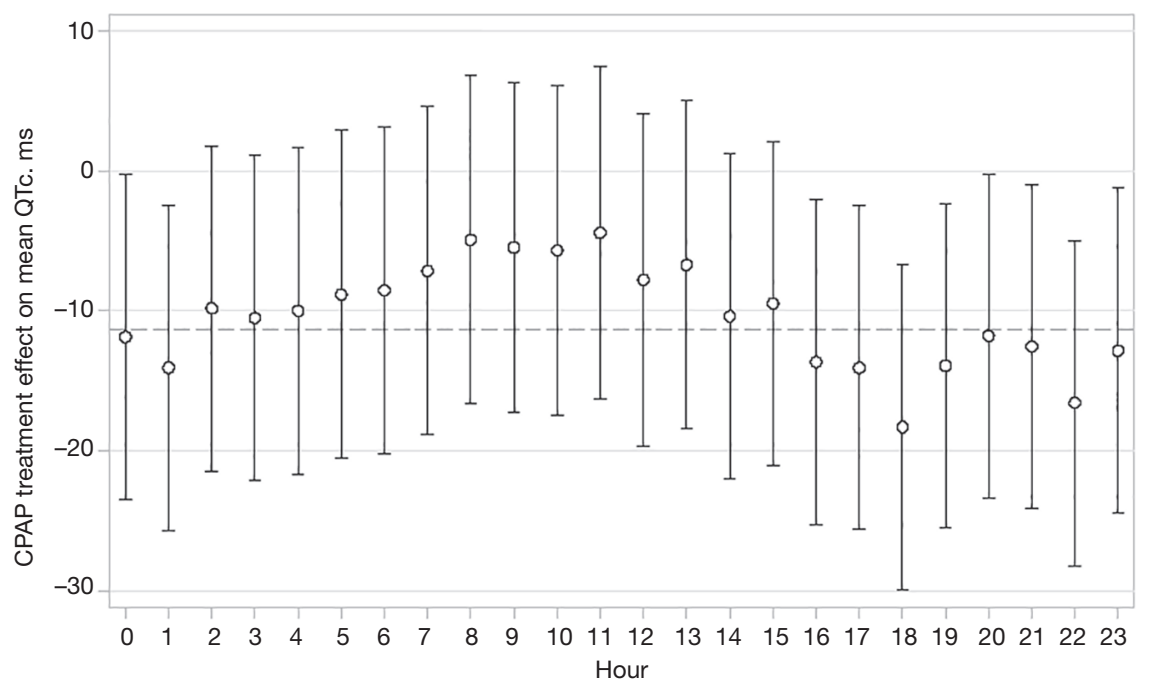

Figure 3 Effect of CPAP on mean QTc in each individual hour. P value for treatment-time interaction: $\mathrm{P}=0.22$. QTc, time interval of cardiac repolarization in $\mathrm{ms}$, corrected for heart rate.

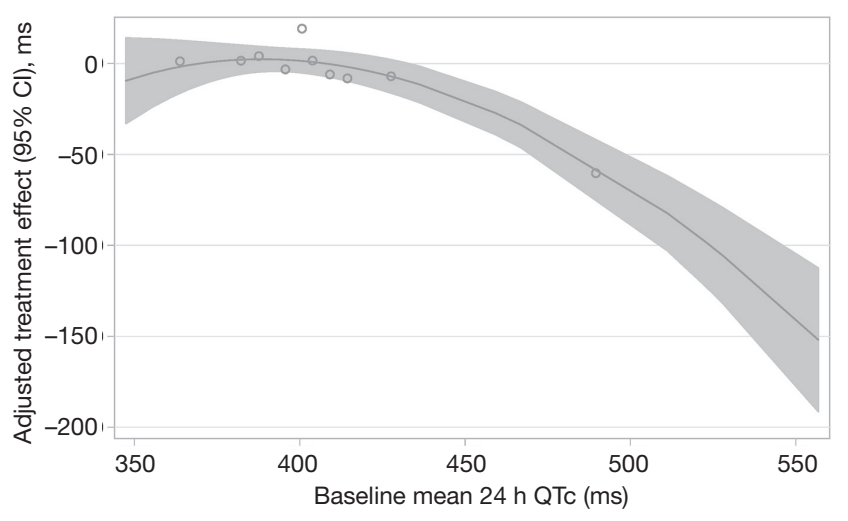

Figure 4 Interaction between (A) the effect of CPAP treatment on mean $24 \mathrm{~h}$ QTc and (B) the mean QTc at baseline. Note that points represent treatment effect estimates in deciles of patients-rather than single individuals_of baseline mean $24 \mathrm{~h}$ QTc. QTc, time interval of cardiac repolarization in $\mathrm{ms}$, corrected for heart rate.

time interaction did not quite reach statistical significance $(\mathrm{P}=0.068)$. Figure 3 shows the CPAP treatment effect on QTc in each individual hour. The CPAP treatment effect on QTc seemed to be mainly driven by 8 patients in the highest QTc decile at baseline (Figure 4) all of whom had a mean baseline $24 \mathrm{~h}-\mathrm{QTc}>430 \mathrm{~ms}$. By contrast, there was no treatment effect in the remaining participants.

Mean $24 \mathrm{~h}$ heart rate decreased by -2.4 beats per minute
(95\% CI, -5.5 to 0.6 ) through use of therapeutic CPAP, when compared with subtherapeutic CPAP, however in this subset of the original trial entrants the reduction was not quite statistically significant $(\mathrm{P}=0.12$; Table 4$)$. No interaction of baseline severity of OSA (ODI) and CPAPcompliance on CPAP treatment effect on 24 h-QTc was found.

\section{Discussion}

The principle findings of this randomized controlled trial are that in patients with moderate-to-severe OSA, treatment with CPAP resulted in significant reductions of the QTcinterval in patients with a high baseline QTc $>430 \mathrm{~ms}$, while no effect of CPAP was observed in patients with shorter QTc-intervals.

Our group previously showed that withdrawal of an established CPAP treatment in moderate-to-severe OSA was associated with considerable prolongations of the QTcand T-peak-to-Tend (TpTe)-intervals (mean difference 21.4 and $14.4 \mathrm{~ms}$, respectively) (7). The current study sought to further investigate CPAP treatment effects on circadian patterns of cardiac repolarization. Contrary to our prior hypothesis, baseline QTc in patients with moderate-tosevere OSA was longest during the evening hours rather than during sleeping hours. This may be because in patients with OSA the sleep period is heavily disrupted and thus interferes with the QTc prolongation with sleep observed 
Table 4 Effect of CPAP on heart rate

\begin{tabular}{|c|c|c|c|c|c|c|}
\hline Time period & \multicolumn{2}{|c|}{ Therapeutic CPAP } & \multicolumn{2}{|c|}{ Subtherapeutic CPAP } & $\begin{array}{l}\text { Treatment effect } \\
(95 \% \mathrm{Cl})\end{array}$ & $P$ value \\
\hline Total $24 \mathrm{~h}$ & $80.7(11.7)$ & $80.2(12.0)$ & $81.8(11.3)$ & $83.5(10.4)$ & $-2.4(-5.5$ to 0.6$)$ & 0.12 \\
\hline $12 \mathrm{pm}-6 \mathrm{am}^{\star}$ & $73.8(12.7)$ & $71.5(12.1)$ & $71.1(10.7)$ & $73.2(10.5)$ & $-0.3(-4.0$ to 3.4$)$ & 0.88 \\
\hline $6 \mathrm{pm}-12 \mathrm{pm}^{*}$ & $87.2(14.1)$ & $85.0(12.0)$ & $82.9(12.8)$ & $87.7(11.6)$ & $-1.6(-5.3$ to 2.1$)$ & 0.39 \\
\hline
\end{tabular}

Data are presented as mean $(\mathrm{SD})$ or mean $(95 \% \mathrm{Cl}) .{ }^{*}$, test for treatment-time interaction: $\mathrm{P}=0.65$.

in normal subjects $(9,10)$. In addition, the treatment effect of CPAP on QTc also seemed to be greatest during the evening hours and not during sleeping hours. This may be because this was the period with the longest QTc at baseline and thus a larger effect size would be expected similar to the phenomenon seen in the 8 patients with the highest baseline QTc. However, it is unclear why a normal pattern of lengthening of QTc during sleep was not fully reestablished. The observed differences in baseline QTc could in part also be due to differences in heart rate at baseline, as both were highest during evening hours. A similar influence of heart rate on the effect of CPAP on QTc seems less likely as CPAP 'normalized' QTc during all time periods, which was not the case with heart rate, where circadian differences largely remained unchanged (see Table 4).

An interesting aspect of our analysis can be seen when comparing QTc-intervals and corresponding standard deviations (SD) in the two groups at follow-up (see Table 3). In the therapeutic CPAP group, irrespective of baseline QTc during all four time periods, inter-individual QTc variation at follow-up was reduced to a rather homogenous low level; in contrast to the subtherapeutic CPAP group, the SD of the mean QTc in the CPAP group is more than halved due to the QTc being 'normalized' in the patients with highest QTc at baseline (Figure 4). One could argue from this finding that CPAP might be able to normalize the QTc-interval, when a certain degree of abnormal prolongation was present before treatment. This argument is reinforced by the observation that the CPAP treatment effect was mainly driven by the 8 patients in the highest decile of baseline QTc (all $>430 \mathrm{~ms}$ ). The greatest CPAP treatment effect on QTc was observed in our previous CPAP withdrawal trial (7), in which patients had higher mean QTc-intervals at baseline when compared with the current trial (419 vs. $408 \mathrm{~ms})$.

\section{Biological plausibility}

The main direct pathophysiological consequences of OSA are intermittent hypoxia, arousals from sleep and intrathoracic pressure swings, all contributing to augmented sympathetic activity (3). Severe OSA has been shown to be associated with decreased baroreflex sensitivity (BRS), a marker of cardiac vagal responsiveness and treatment with CPAP in moderate-to-severe OSA increased daytime BRS by $24 \%$ after 4 weeks (14). This improvement is comparable with the effect of several months of endurance training in patients with mild hypertension (15). In the study by Kohler et al. (14) urine normetanephrine excretion decreased by $26 \%$ with CPAP treatment. These findings all suggest that CPAP therapy in moderate-to-severe OSA patients decreases sympathetic overstimulation and improves sympathico-vagal imbalances.

We have previously shown, in a model simulating OSA in 44 patients with paroxysmal atrial fibrillation, a mean QTc prolongation at the end of a $20 \mathrm{~s}$ simulated obstructive apnoea of $17.3 \mathrm{~ms}$ (95\% CI, 10.7 to $23.9 \mathrm{~ms}$, $\mathrm{P}=0.001$ ) when compared with normal breathing (16). The timing and magnitude of the QTc-prolongation was strongly correlated with heart rate increases in the latter study suggesting the importance of sympathetic activation. A rise of over $200 \%$ in sympathetic activity was also found by Somers et al. (17) at the end of 20 seconds simulated apnoea. Although these conclusions derive from indirect observations of cardiac autonomic nerve activity, they imply pathophysiological OSA consequences that could lead to a prolongation of the QTc-interval and the reversal through use of CPAP therapy. 


\section{Impact of QTc on SCD?}

While the effect of QTc-prolongation on the incidence of SCD in the general population seems to be small and difficult to detect reliably, the effect in patients with underlying cardiovascular disease is likely to be greater, as pointed out by Montanez et al. (18). In a case-control study by Panikkath et al. (19), QTc was the only ECG-derived interval independently associated with SCD in patients with left ventricular hypertrophy. The patients in our study had a relatively high cardiovascular risk profile, typical for the majority of patients diagnosed with moderate-to-severe OSA. In the Rotterdam QT project, a QTc-interval $>440$ ms was associated with a 2.3-times higher risk for SCD when compared with a QTc $\leq 440 \mathrm{~ms}$ (95\% CI, 1.4-3.9) (20). Taken together, OSA seems capable of increasing, and CPAP seems capable of reducing, QTc in OSA patients with high baseline QTc, and thus decreasing risk for SCD.

\section{Strengths and limitations}

Many studies on repolarization changes are based on a onetime analysis of standard 12-lead-ECGs and thus are prone to measurement-errors and in case of non-randomized studies, to type I error. In the current study, we used 24 h-Holter-ECG data and calculated weighted means of all QTc-values, making measurement errors negligible. However, we analysed data from a previously published randomized trial, thus our interpretations must be regarded as hypothesis generating rather than fully robust.

\section{Conclusions}

CPAP seems capable of improving an elevated QTc-interval, a biomarker for SCD, in patients with moderate-to-severe OSA. In selected patients, treatment with CPAP could, in addition to beneficial effects on systolic and diastolic blood pressure, possibly reduce the incidence of SCD. Future randomized trials could investigate the effect of CPAP on repolarization in moderate-to-severe OSA patients with prolonged QTc.

\section{Acknowledgements}

None.

\section{Footnote}

Conflicts of Interest: Prof. Stradling reports personal fees from ResMed UK, outside the submitted work. Prof. Kohler reports grants from University of Zurich during the conduct of the study. Other authors have no conflicts of interest to declare.

Ethical Statement: The trial from which the data were drawn was previously published in 2002 and was approved by the central Oxford research ethics committee (Oxford, UK; COREC No. 96.127).

\section{References}

1. Heinzer R, Vat S, Marques-Vidal P, et al. Prevalence of sleep-disordered breathing in the general population: the HypnoLaus study. Lancet Respir Med 2015;3:310-8.

2. Young T, Peppard PE, Gottlieb DJ. Epidemiology of obstructive sleep apnea: a population health perspective. Am J Respir Crit Care Med 2002;165:1217-39.

3. Kohler M, Stradling JR. Mechanisms of vascular damage in obstructive sleep apnea. Nat Rev Cardiol 2010;7:677-85 .

4. Gami AS, Olson EJ, Shen WK, et al. Obstructive sleep apnea and the risk of sudden cardiac death: a longitudinal study of 10,701 adults. J Am Coll Cardiol 2013;62:610-6.

5. Goldberger JJ, Basu A, Boineau R, et al. Risk stratification for sudden cardiac death: a plan for the future. Circulation 2014;129:516-26.

6. Gami AS, Howard DE, Olson EJ, et al. Day-night pattern of sudden death in obstructive sleep apnea. N Engl J Med 2005;352:1206-14.

7. Rossi VA, Stoewhas AC, Camen G, et al. The effects of continuous positive airway pressure therapy withdrawal on cardiac repolarization: data from a randomized controlled trial. Eur Heart J 2012;33:2206-12.

8. Pepperell JC, Ramdassingh-Dow S, Crosthwaite N, et al. Ambulatory blood pressure after therapeutic and subtherapeutic nasal continuous positive airway pressure for obstructive sleep apnoea: a randomised parallel trial. Lancet 2002;359:204-10.

9. Molnar J, Zhang F, Weiss J, et al. Diurnal pattern of QTc interval: how long is prolonged? Possible relation to circadian triggers of cardiovascular events. J Am Coll Cardiol 1996;27:76-83.

10. Bonnemeier H, Wiegand UK, Braasch W, et al. Circadian profile of QT interval and QT interval variability in 172 healthy volunteers. Pacing Clin Electrophysiol 2003;26:377-82.

11. Johns MW. A new method for measuring daytime sleepiness: the Epworth sleepiness scale. Sleep 
1991;14:540-5.

12. Bazett HC. An analysis of time-relations of electrocardiograms. Heart 1920:353-7.

13. Royston P, Sauerbrei W. A new approach to modelling interactions between treatment and continuous covariates in clinical trials by using fractional polynomials. Stat Med 2004;23:2509-25.

14. Kohler M, Pepperell JC, Casadei B, et al. CPAP and measures of cardiovascular risk in males with OSAS. Eur Respir J 2008;32:1488-96.

15. Somers VK, Conway J, Johnston J, et al. Effects of endurance training on baroreflex sensitivity and blood pressure in borderline hypertension. Lancet 1991;337:1363-8.

16. Schlatzer C, Schwarz EI, Sievi NA, et al. Intrathoracic pressure swings induced by simulated obstructive sleep apnoea promote arrhythmias in paroxysmal atrial fibrillation. Europace 2016;18:64-70.

Cite this article as: Schlatzer C, Bratton DJ, Schwarz EI, Gaisl T, Pepperell JC, Stradling JR, Kohler M. Effect of continuous positive airway pressure therapy on circadian patterns of cardiac repolarization in patients with obstructive sleep apnoea: data from a randomized trial. J Thorac Dis 2018;10(8):4940-4948. doi: $10.21037 /$ jtd.2018.07.17
17. Somers VK, Dyken ME, Skinner JL. Autonomic and hemodynamic responses and interactions during the Mueller maneuver in humans. J Auton Nerv Syst 1993;44:253-9.

18. Montanez A, Ruskin JN, Hebert PR, et al. Prolonged QTc interval and risks of total and cardiovascular mortality and sudden death in the general population: a review and qualitative overview of the prospective cohort studies. Arch Intern Med 2004;164:943-8.

19. Panikkath R, Reinier K, Uy-Evanado A, et al. Electrocardiographic predictors of sudden cardiac death in patients with left ventricular hypertrophy. Ann Noninvasive Electrocardiol 2013;18:225-9.

20. Algra A, Tijssen JG, Roelandt JR, et al. QTc prolongation measured by standard 12-lead electrocardiography is an independent risk factor for sudden death due to cardiac arrest. Circulation 1991;83:1888-94. 